\title{
Implications of a temperature-dependent magnetic anisotropy for superparamagnetic switching
}

\author{
Martin Stier ${ }^{\mathrm{a}}$, Alexander Neumann ${ }^{\mathrm{b}}$, André Philippi-Kobss ${ }^{\mathrm{b}, \mathrm{c}}$, Hans Peter \\ Oepen $^{\mathrm{b}}$, Michael Thorwart ${ }^{\mathrm{a}}$ \\ ${ }^{a}$ I. Institut für Theoretische Physik, Universität Hamburg, Jungiusstraße 9, 20355 \\ Hamburg, Germany \\ ${ }^{b}$ Institut für Nanostruktur- und Festkörperphysik, Universität Hamburg, Jungiusstraße \\ 11, 20355 Hamburg, Germany \\ ${ }^{c}$ Deutsches Elektronen-Synchrotron DESY, Notkestr. 85, 22607 Hamburg, Germany
}

\begin{abstract}
The macroscopic magnetic moment of a superparamagnetic system has to overcome an energy barrier in order to switch its direction. This barrier is formed by magnetic anisotropies in the material and may be surmounted typically after $10^{9}-10^{12}$ attempts per second by thermal fluctuations. In a first step, the associated switching rate may be described by a Néel-BrownArrhenius law, in which the energy barrier is assumed as constant for a given temperature. Yet, magnetic anisotropies in general depend on temperature themselves which is known to modify the Néel-Brown-Arrhenius law. We illustrate quantitatively the implications of a temperature-dependent anisotropy on the switching rate and in particular for the interpretation of the prefactor as an attempt frequency. In particular, we show that realistic numbers for the attempt frequency are obtained when the temperature dependence of the anisotropy is taken into account.
\end{abstract}

Keywords: Superparamagnetism, Arrhenius law, Néel-Brown theory, attempt frequency

\section{Introduction}

The development of devices for magnetic storage media faces several challenges in the ongoing miniaturization of information units. One of the fundamental physical problems is the so-called superparamagnetic limit. In the small scale limit, the alignment of the macroscopic magnetic moment of the 
ferromagnetic particle with a direction along a preferred axis is no longer stable, but permanently affected by thermal fluctuations. Despite similarities between para- and superparamagnetism, distinct differences exist. Quantum mechanics allows us to describe paramagnetism rigorously with the magnetic field treated as a small perturbation. In superparamagnetism, the spins of a nanostructure are coupled by the exchange interaction which causes a quasiclassical behavior of a collective magnetic moment with a large variety of possible energetically continuous states. Due to the large number of coupled spins which form the macroscopic magnetic moment, smaller contributions of the single spins to the total energy may become important. They can be rooted in, e.g., dipolar and/or spin-orbit interaction effects. The collection of such secondary effects is commonly summarized to form the anisotropies. The energy scale of a nanomagnet with its small dimensions can become comparable with the thermal energy. The total energy of the nanomagnet with its collectively formed magnetic moment reveals remarkable differences compared to the case of a single moment. In particular, due to the competition with thermal energies, important collective features strongly change as function of temperature. For example, the blocking of the macrospin along a certain direction (easy axis of magnetization) is substantially influenced. Here, two energetically degenerate states are oriented parallel to the easy axis and are separated by an energy barrier $\Delta E$. The latter suppresses thermal switching. In the case of a uniaxial system, the barrier height scales with the volume $V$ and strength of the anisotropy $K$. At high enough temperature, the barrier can be overcome and the collective magnetic moment can flip back and forth, resulting in a vanishing time averaged magnetic moment. In superparamagnetic systems, a problem becomes immediately obvious since temperature determines both the collective magnetization as well as the switching rate. This is in the focus of the present work.

Superparamagnetic behavior of nanomagnets is usually analyzed in terms of the switching of the magnetization [1, 2]. To this end, a switching frequency $f(T)$ is determined as a function of temperature $T$ which commonly reveals an Arrhenius-like temperature dependence following

$$
f(T)=f_{0} e^{-\frac{K V}{k_{B} T}}
$$

with $f_{0}$ being the so-called attempt frequency and $k_{B}$ the Boltzmann constant. To obtain an Arrhenius law, a temperature-independent anisotropy energy has to be assumed, such that a separation of time scales is possible [5]. 
Thus, the switching events have to be rare compared to the frequent attempts made until switching occurs, meaning $f(T) \ll f_{0}$ or $K V \gg k_{B} T$, respectively. When the logarithm of the temperature-dependent switching frequency is plotted versus the inverse temperature, a straight line results, with the slope yielding the anisotropy energy and the intercept yielding the attempt frequency. Such an analysis is common to many experiments and is appealing due to its simplicity, albeit detailed investigations whether a clear separation of time scales is really given are not properly made and are sometimes not even possible due to experimental constraints.

Even though the magnitude and the dependence on temperature of the attempt frequency have been modeled on the basis of detailed assumptions about the reversal mechanisms [3, 4, 5, 6, 7], large deviations from these predictions by several orders of magnitude are reported in the literature [8, 9, 10]. Several possible physical explanations for these substantial discrepancies have been offered since then [1, 11, 12].

In this work, we readdress these discrepancies by analyzing results from such an Arrhenius fit. Certainly, the Arrhenius plot itself is a powerful tool and its validity is confirmed by innumerable successful applications. The mere numbers that come out of such an analysis are correct on their own, but point out that the assignment to specific material properties has to be done with care. In particular, precise knowledge about the temperature dependence of the exponent in Eq. (1) is necessary for reliable extractions of material parameters. Likewise, a straightforward extrapolation of the Arrhenius plot to zero temperature in order to derive the prefactor $f_{0}$ and interpret it as a constant "attempt frequency" has to be done with caution.

In this context, it was shown that the use of the free energy instead of the total energy entering for the energy barrier generates a temperature dependent contribution in connection with the entropy [13, 14]. This additional contribution to the activation energy can reduce the prefactor [15] compared to Brown's result [3] and plays an important role for large statistical ensembles with a broad distribution of activation energies and high-dimensional energy landscapes [15].

Of more importance is the explicit dependence of the anisotropy on temperature, as addressed in the present work in greater detail. This has been investigated mostly in the blocked regime where switching is largely suppressed [16, 17, 18, 19], but has also been mentioned to influence the superparamagnetic switching [16, 20].

There are several origins of a temperature dependence of the total anisotropy. 
At first, the magnetic anisotropy can vary with temperature due to slight changes of the structure and stress in the material. This is well known for bulk materials like Co. Second, the effect can be due to the shape anisotropy which is often the origin of the uniaxial anisotropy of nanoparticles. As this part is determined by the saturation magnetization, it changes with varying temperature. The third influence originates from the scaling property of the magneto-crystalline anisotropy with the saturation magnetization in a powerlaw like characteristics [21, 22, 23, 24, 25, 26]. The temperature dependence of the effective anisotropies in magnetic nanoparticles with different shapes with cubic or uniaxial bulk anisotropy and Néel surface anisotropy has been calculated by using a constrained Monte Carlo approach [27]. The impact of thermal magnon excitations on coercivity has been investigated in Ref. [28].

The effect of the temperature dependence of the shape anisotropy on the coercivity for aligned Stoner-Wohlfarth systems has been considered in Ref. [19]. The standard Néel-Brown formula for the coercive field has been extended to include the temperature dependence of the magnetization, leading to an effective temperature dependent anisoptropy barrier. The role of a temperature dependent magnetocrystalline anisotropy on the coercivity of nanostructured materials was investigated theoretically in Ref. [16]. Furthermore, it was shown in Ref. [29] that the temperature dependence of the magnetic anisotropy also needs to be carefully taken into account when the magnetic remanence is considered.

Experimental results on Co-Fe magnetic nanoparticles [30] show that the temperature dependence of the anisotropy has to be taken into account for a matching with a Néel-Brown-Arrhenius law. In this case, an empirical Brükhatov-Kirensky ansatz was used for the temperature dependence of the anisotropy. Such an approach reproduces realistic zero-temperature values $K(0)$ for the bulk anisotropies as well as realistic times for the inverse attempt frequencies.

The purpose of this work is not to address the details of the physical effects, which contribute to the temperature dependence of the anisotropy. Instead, our focus is on its general impact on the analysis of the superparamagnetic behavior via its switching characteristics. We demonstrate that the temperature interval (which is usually somewhat limited in experiments) in which a finite number of data points are fitted to an Arrhenius law has to be selected with care. It may determine very sensitively the resulting parameters of the Arrhenius plot, being the slope (anisotropy) and the intercept (attempt frequency). In general, besides the blocking temperature $T_{B}$ 


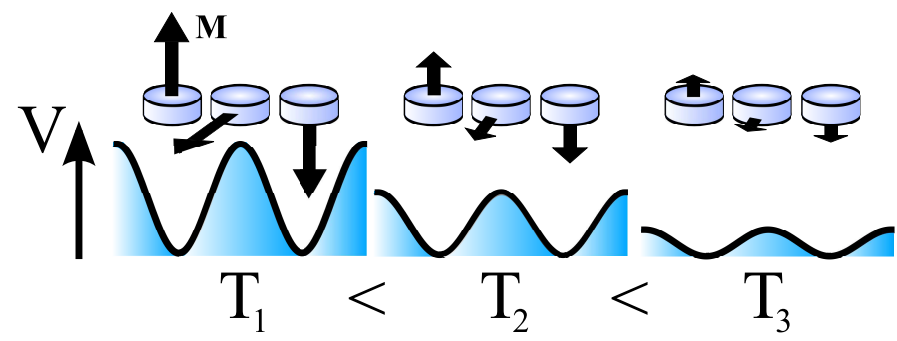

Figure 1: Sketch of magnetic potentials of superparamagnetic nanomagnets for different temperatures with the easy axis pointing in the out-of-plane direction. To switch between the minima, the magnetization $\mathbf{M}$ has to overcome the anisotropy energy barrier which typically depends on $\mathbf{M}(T)$. With increasing temperature, both the magnetization and the energy barrier, are decreased.

in superparamagnetism, a second temperature scale becomes relevant in the macrospin description, i.e., the Curie temperature $T_{C}$, being decisive for the magnetic ordering. Thus, experimentally determined prefactors [8, 31, 32, 33] have to be carefully interpreted, particularly, but not exclusively, when the temperature range, in which the switching measurement are performed, is comparable to the magnetic ordering temperature [16]. We illustrate quantitatively that naively assuming the applicability of the Arrhenius law may give rise to unintended misinterpretations if a temperature-independent height of the energy barrier is presumed.

\section{Theoretical models for a temperature-dependent anisotropy}

A generic superparamagnetic system can be described by the Heisenberg model

$$
H=-J \sum_{\langle i, j\rangle} \mathbf{S}_{i} \cdot \mathbf{S}_{j}+H_{\text {aniso }},
$$

where the spins $\mathbf{S}_{i}$ tend to align parallel due to the exchange interaction of strength $J$ in order to form a macroscopic magnetic moment. The exchange interaction itself is rotationally invariant and thus does not favor a certain direction for the resulting magnetic moment. It basically determines the temperature dependence of the bulk magnetic system. However, superparamagnetic systems typically possess either intrinsic material symmetries or external ones such as a shape anisotropy. Then, distinct directions of the magnetic moment are preferred. To model this feature, a generic anisotropy term $H_{\text {aniso }}$ is introduced in Eq. (2). The specific form of the anisotropy has 
to be defined for the particular experimental situation. It is important to realize that the anisotropy part in small systems does not only define easy or hard axes, but also influences the temperature dependence of the magnetization $M(T)$ itself. Thus, $M(T)$ in small systems can be very different from comparable bulk systems. The theoretical determination of $M(T)$ in those small samples is a non-trivial task [13] and depends on the details of the Heisenberg model. As a matter of fact, we focus on general consequences of a temperature dependence of the magnetization and we do not aim to calculate $M(T)$ from first principles. For the purpose of this work, it is sufficient to assign some generic behavior to it. First, we assume the magnetic system to be large enough to form a collective macroscopic magnetic moment but still sufficiently small to avoid a separation into multiple magnetic domains. In this superparamagnetic limit, we can replace the collection of microscopic quantum spins by a classical magnetic moment $\mathbf{M}$. Changing to the magnetization as the order parameter, the uniaxial anisotropy is described by an energy density. The anisotropy part in Eq. (2) becomes

$$
H_{\text {aniso }}=-K V \cos ^{2} \theta
$$

with $\theta$ being the angle which the magnetization encloses with the z-axis. The total Hamiltonian for a particle of volume $\mathrm{V}$ and with the magnetization $\mathbf{M}$ has two energy minima for $\mathbf{M}= \pm|\mathbf{M}| \mathbf{e}_{z}$. In order to reverse its direction, the total magnetic moment has to switch from one minimum to the other by surmounting the energy barrier $\Delta E=H_{\text {aniso }}\left(\theta=90^{\circ}\right)-H_{\text {aniso }}\left(\theta=0^{\circ}, 180^{\circ}\right)=$ $K V$ while crossing the highest energy state at $\theta=90^{\circ}$. The interaction part of Eq. (2) is irrelevant for determining the energy barrier as it is rotationally invariant and will only be considered implicitly as the mechanism causing the magnetic order. At finite temperature, the difference of the free energy $\Delta F(T)=\Delta E(T)-T \Delta S(T)$ determines the height of the switching barrier. However, we assume that the entropy $S(T)$ does not change significantly by a rotation of the magnetization and set $T \Delta S \ll \Delta E[34]$.

For the following discussion, it is crucial to note that the anisotropy is related to the particle magnetization via a conventional power law $K=$ $\tilde{K}[M(T) / M(0)]^{\nu}$ with the temperature dependent magnetization $M(T)$ and $M(0)$ and $\tilde{K}$ being the corresponding values at zero Kelvin [13, 24, 34, 35, 36. For the sake of concreteness, we consider the case where the uniaxial anisotropy is of dipolar origin. Then, the energy difference between hard and easy axis is given by $\Delta E=\Delta N V M^{2}$ [37] with $\Delta N$ stemming from the 


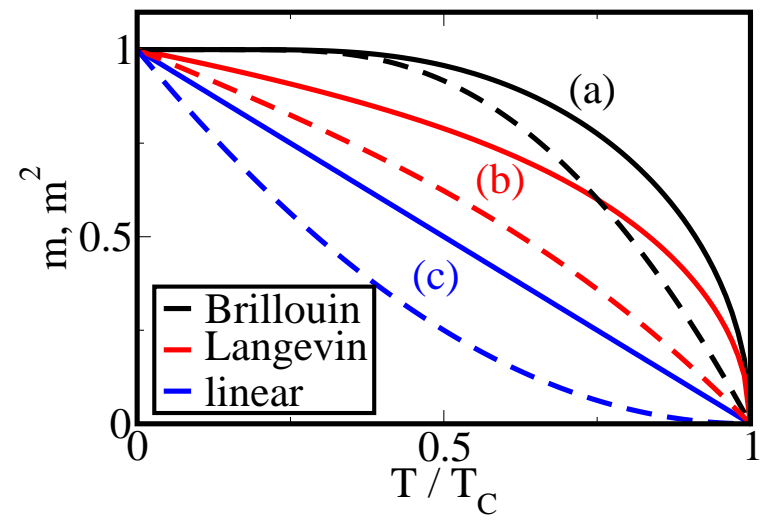

Figure 2: Temperature dependence of the magnetization $m=M(T) / M(0)$ (full lines) and its square $m^{2}$ (dashed lines) described by (a) the Brillouin function $B\left(S, T_{C}\right)$ for a spin $S=1 / 2$, (b) the Langevin function $L\left(T_{C}\right)$ for a classical spin $(S \rightarrow \infty)$, and (c) a linear dependence $m(T)=1-T / T_{C}$. $T_{C}$ denotes the Curie temperature of the system.

demagnetization tensor $\hat{N}$. Thus, we find

$$
\Delta E(T)=\Delta N V M(T)^{2} \equiv K_{0} M(T)^{2},
$$

with $\Delta E(T)$ typically decreasing with increasing temperature. This is schematically shown in Fig. 1.

\section{Results and discussion}

\subsection{Generalized Néel-Brown-Arrhenius law}

Using this model of a temperature dependent energy barrier, we next study the implication on the switching rate of Eq. (11). Therefore, we insert Eq. (4) into Eq. (11) and find a generalized Néel-Brown-Arrhenius law

$$
f(T)=f_{0} e^{-\frac{K_{0} M(T)^{2}}{k_{B} T}} .
$$

In the following, we consider three different conventional model systems yielding a particular $M(T)$ which actually follow this law and, most importantly, investigate the consequences of treating the switching dynamics on the basis of a "conventional" Arrhenius law with a temperature-independent energy barrier, as it is often done in the analysis of experimental data. To fix the time scale, we set the attempt frequency at a typical value of $f_{0}=10^{9} \mathrm{~s}^{-1}$ and choose the anisotropy energy as $K_{0} M(T=0)^{2}=1 \mathrm{eV}$ throughout this work. 


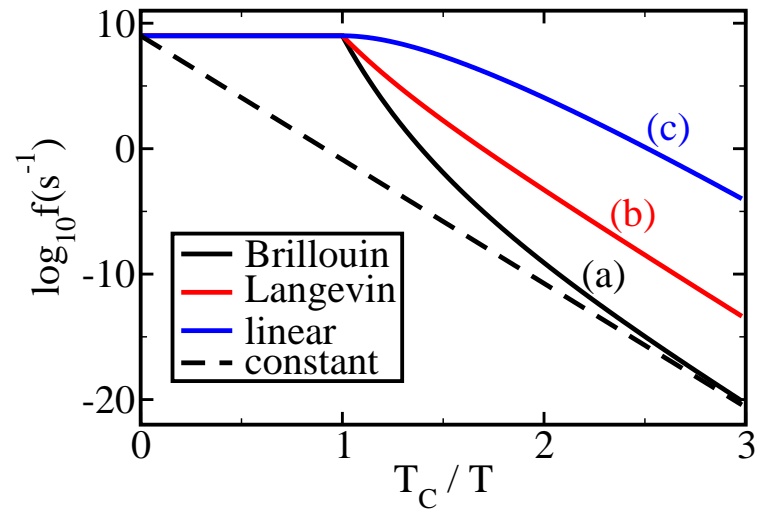

Figure 3: Decadic logarithm of the switching rate Eq. (5) for the temperature-dependent magnetizations shown in Fig. 2. Strong deviations from an Arrhenius law with a constant anisotropy $K_{0} M(T)^{2} \equiv K_{0} M(0)^{2}$ (dashed line) appear even for $T \ll T_{C}$. Parameters are $f_{0}=10^{9} \mathrm{~s}^{-1}$ and $K_{0} M(0)^{2}=1 \mathrm{eV}$.

The conclusions below hold even for reasonable deviations from Eq. (5). For example, a different exponent $\nu$ in $M(T)^{\nu}$ may be absorbed in the actual temperature dependence of $M(T)$, while a (non-exponential) temperature dependence of the prefactor $f_{0}[6]$ is in first order negligible.

The actual magnetization of a physical system is affected by a multitude of material properties, such as intrinsic interactions or the finite sample size. While in ferromagnetic bulk samples the temperature dependence of the magnetization is typically well described by a Brillouin function, this is often not true in systems of reduced dimensions [36, 38, 39]. Thus, an assumption on the general form of $M(T)$ cannot be made without explicitly knowing the specific system. Yet, to illustrate that the explicit temperature-dependence of $M(T)$ has a major effect on the switching rate, we consider three different examples in this work: (a) the Brillouin function describes quantum spins in a bulk sample, (b) the Langevin function refers to a classical magnetic moment, and (c) a linear dependence such that $M(T)=M(0)\left(1-T / T_{C}\right)$ (with the Curie temperature $T_{C}$ ) as a possible variant of an unconventional magnetization curve which can be realized in magnetic nano-islands [13]. These functions and their squares are shown in Fig. 2,

In general, $M(T)$ decreases with increasing temperature and vanishes at the Curie temperature $T_{C}$ which also holds for $\Delta E(T) \propto M(T)^{2}$. Thus, the system does not only switch faster with growing temperature due to an explicit increase of thermal fluctuations, but also due to a lower anisotropy 
barrier. This has a pronounced effect on the switching rate which is shown in Fig. 3. For convenience we use the decadic logarithm lg $\equiv \log _{10}$ to directly determine the exponent $y$ of the frequency according to $f=10^{y} \mathrm{~s}^{-1}$. Strong deviations from an Arrhenius law with a constant energy barrier are clearly visible.

These deviations are not captured by a linear adjustment of the Arrhenius law with a temperature shift $T \rightarrow T-T_{0}$, which yields to the Vogel-Fulcher law. Above $T_{C}$, this is obvious since the magnetization, and with it the energy barrier, vanishes. Close to $T_{C}$, the explicit values of $f\left(T \approx T_{C}\right)$ lose their physical meaning since the concept of a switching rate with a separation of time scales breaks down [7]. However, from the existence of a finite critical temperature $T_{C}$ with $\Delta E\left(T \geq T_{C}\right) \equiv 0$ and $f\left(T \geq T_{C}\right) \equiv f_{0}$, we have to conclude that such a system cannot be described by a constant energy barrier as in this case $f_{\Delta E=\text { const }}(T)$ would approach $f_{0}$ only for $T \rightarrow \infty$. Thus, an accurate description of the switching rate requires in principle the knowledge of the full temperature-dependent magnetization $M(T)$ or, more precisely, of the energy barrier $\Delta E(T)$.

\subsection{Interpretation of the prefactor}

The generalized Néel-Brown theory of the switching rate has profound consequences for the interpretation of experimental data. In particular, the interpretation of the prefactor in terms of an attempt frequency has to be revisited. In real systems, it is not a realistic task to measure the switching rate $f(T)$ over the entire temperature regime from $T=0$ to $T_{C}$. Usually, only a finite (and small) temperature interval $\left[T_{\min }, T_{\max }\right]$, with $\Delta T=T_{\max }-$ $T_{\min } \approx 30 \ldots 50 \mathrm{~K}[8,32$ ] is experimentally accessible. Outside of this range, the switching is either too fast to be observable with present day tools or too slow to be measured in a reasonable time. In fact, $\Delta T$ is usually small enough that the magnetization does not significantly change with temperature in this selected temperature window and the switching rate appears to be linearly dependent on $T^{-1}$ in this small interval. Thus, it is tempting to fit the results to a linear function

$$
\lg f=\lg f_{0}^{\mathrm{fit}}-\lg (e) \frac{K_{0}^{\mathrm{fit}}}{k_{B} T}
$$

and to determine $f_{0}$ and $K_{0}$. However, the interpretation of the results for the fitted parameters $f_{0}^{\text {fit }}$ and $K_{0}^{\text {fit }}$ can actually be misleading. We illustrate this for a simple analytical example for which we assume at this point a temperature-dependent magnetization $M(T)=M(0) \sqrt{1-T / T_{C}}$. 
The switching rate resulting from Eq. (5) is now given by $\lg f=\lg f_{0}^{\text {fit }}-$ $\lg (e)\left(K_{0} M(0)^{2}\right) /\left(k_{B} T\right)$ with $\lg f_{0}^{\text {fit }}=\lg f_{0}+\lg (e)\left(K_{0} M(0)^{2}\right) /\left(k_{B} T_{C}\right)$. This equation is in fact linearly depending on $1 / T$ and would actually yield the result for $K_{0}^{\text {fit }}=K_{0} M(0)^{2}$. However, more importantly, the exponent of the fitted prefactor (which is often interpreted as the attempt frequency) differs from the physical one by $\lg (e) K_{0} M(0)^{2} /\left(k_{B} T_{C}\right)$. This difference can be very pronounced. For exapmle if $f_{0}=10^{9} \mathrm{~s}^{-1}$ and $K_{0} M^{2}(0)=1 \mathrm{eV}$ then $\lg f_{0}^{\text {fit }} \approx \lg f_{0}+\frac{5000 \mathrm{~K}}{T_{C}}$. Hence, the attempt frequency $f_{0}=10^{y}$ is in this case is overestimated by five orders of magnitude for a typical $T_{C}$ of $1000 \mathrm{~K}$.

For the three cases of $M(T)$ used above, the deviation of the prefactor becomes also temperature dependent now (this is not the case for the simplified model used in the above paragraph). This effect can be very pronounced. To show this, we use the same model parameters as above and numerically perform linear fits according to Eq. (6) for a given small temperature interval $T \in\left[T^{\mathrm{fit}}-T_{C} / 200, T^{\mathrm{fit}}\right]$ in order to mimic the situation of typical experiments. The results are shown in Fig. 4. The linear fits in general overestimate $f_{0}$ by several orders of magnitude. Interpreted as attempt frequencies, such linear fits yield unrealistically large values. This explains the unphysical values of the attempt frequencies reported in the analysis of experimental data on the basis of an Arrhenius or a Vogel-Fulcher law [8, 31, 32, 33]. Naturally, the fitted values of $f_{0}$ are more accurate when the magnetization changes only very little in the respective temperature interval used for the fit. Then, the magnetization is well approximated by the constant $K_{0}^{\text {fit }}$. For a Brillouin function, the (squared) magnetization varies less at lower temperatures (cf. Fig. (2). Thus, the best fits are achieved for very low temperature. On the contrary, $m(T)^{2}=\left[1-\left(T / T_{C}\right)\right]^{2}$ changes the least close to the Curie temperature, which is also reflected in Fig. (4. Obviously, fits with the assumption of a constant energy barrier cannot deliver reliable results for $K_{0}$, when $\Delta E(T)$ is actually changing with $T$. Even though $K_{0}$ probably is not misdetermined by orders of magnitude as it is fitted directly (and not its logarithm at $T \rightarrow \infty$ as for $f_{0}$ ), the correction of the barrier height has to be taken into account as well.

The important consequences of a finite temperature window chosen for the analysis for the exponent of the fitted prefactor are illustrated in more detail in Fig. 5. We vary the borders of the temperature window used for the fits and also the Curie temperature. In general, the regions for the optimal temperature $T^{\mathrm{fit}}$ are confirmed. An almost temperature-independent magnetization yields a well fitted attempt frequency $f_{0}^{\text {fit }} \approx f_{0}=10^{9} \mathrm{~s}^{-1}$. Ev- 


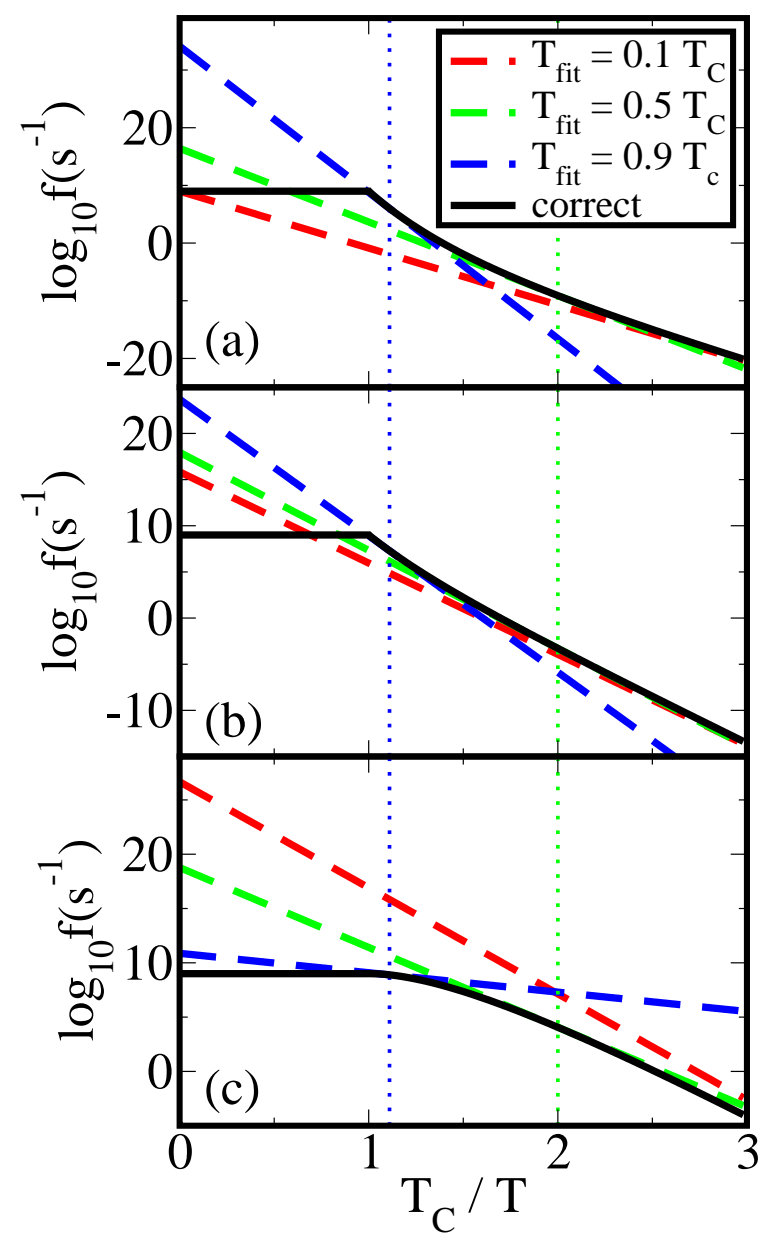

Figure 4: Linear fits (dashed lines) to the switching rate $\lg f=\lg f_{0}-$ $\lg (e) K_{0} M(T)^{2} /\left(k_{B} T\right)$ (full line), performed in a finite temperature interval around the given $T_{\text {fit }}$ indicated by the vertical dotted lines (except for $T_{\text {fit }}=0.1 T_{C}$ which lies out of bounds) for (a) a Brillouin function for $S=1 / 2$, (b) a Langevin function, and (c) a linear dependence (cf. Fig. 22). The exponents of $f_{0}^{\text {fit }}=f$ fit $\left(T_{C} / T \rightarrow 0\right)$ can differ from the actual $f_{0}$ by several orders of magnitude. The parameters are $f_{0}=10^{9} \mathrm{~s}^{-1}, K_{0} / M(0)^{2}=1 \mathrm{eV}$ and $T_{C}=500 \mathrm{~K}$.

erywhere else away from this optimal value, it is significantly overestimated. Moreover, the lower the Curie temperature is, the worse are the fitted results, which can be again traced back to the temperature dependence of $M(T)$. As the derivative $\partial_{\tilde{T}} M^{2}(\tilde{T})$ with respect to $\tilde{T}=T / T_{C}$ remains constant for all $T_{C}$ for the three cases treated here, the derivative $\partial_{T} M^{2}(\tilde{T})=T_{C}^{-1} \partial_{\tilde{T}} M^{2}(\tilde{T})$ 

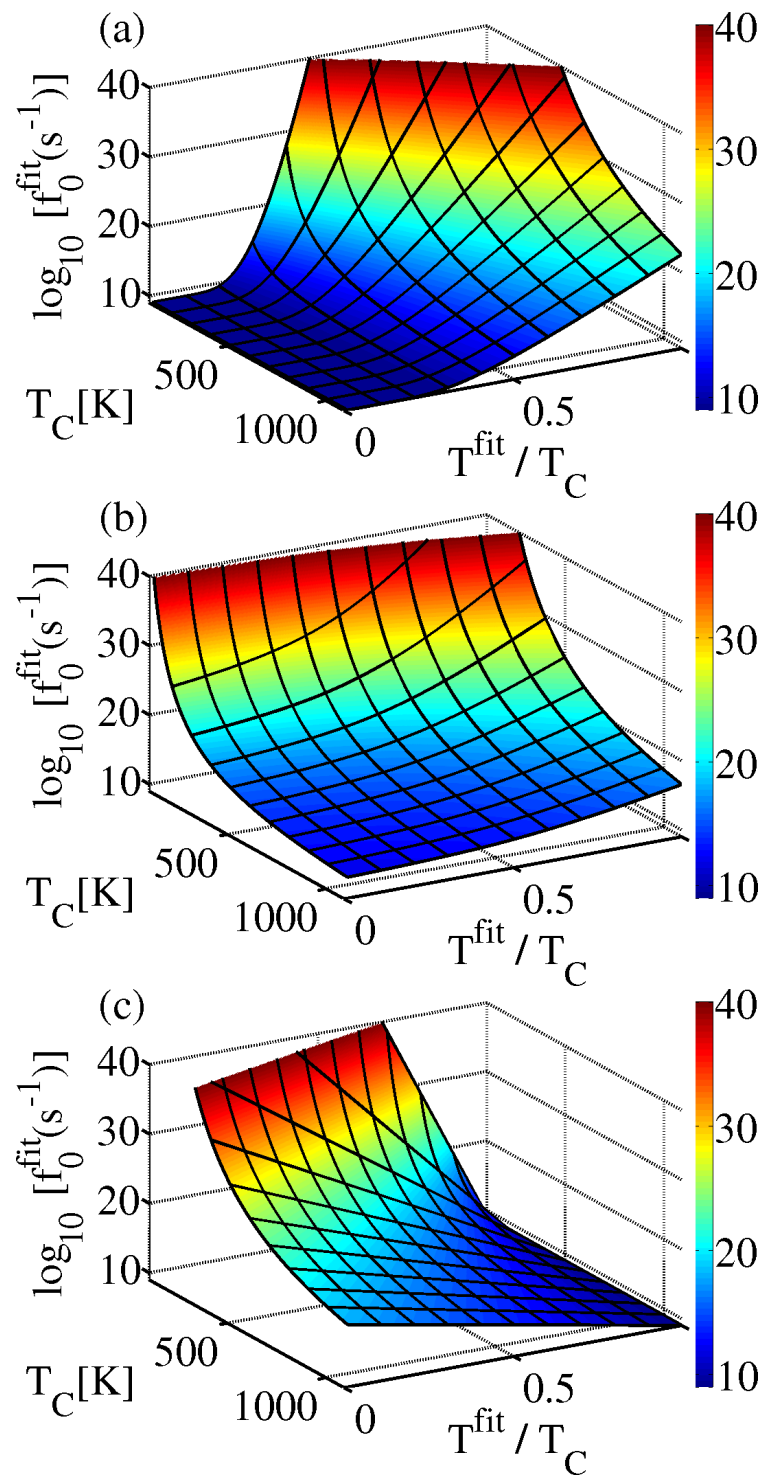

Figure 5: Fitted exponent of the attempt frequency. The magnetization is calculated by (a) a Brillouin function for $S=1 / 2$, (b) the Langevin function (classical magnetic moment) and (c) by a linear approach (cf. Fig. 2). The correct exponent would be $\lg f_{0}\left(\mathrm{~s}^{-1}\right)=9$.

is proportional to the inverse Curie temperature. Thus, we find a larger deviation for a lower $T_{C}$. This is important for the experimental analysis since superparamagnetic phenomena are typically investigated for very small samples where the Curie temperature may be notably smaller than in a bulk 
sample [40].

\section{Conclusions}

In this work we have illustrated quantitatively that a temperature-dependent magnetic anisotropy in a superparamagnetic system can give rise to significant corrections to the standard Néel-Brown-Arrhenius law with a temperature independent energy barrier. For a correct interpretation of the parameters, the energy barrier for the switching behavior of the collective magnetic moment has to be based on a temperature-dependent magnetic anisotropy even in a simple model. This generates corrections to the Néel-BrownArrhenius law. In particular, the prefactor can no longer be determined in general by a fit of measured data on the basis of a temperature-independent anisotropy. Even if the fit appears plausible in a given finite temperature range where a seemingly linear dependence of $f$ on $1 / T$ is found, the attempt frequency can be incorrectly determined by several orders of magnitude.

Although the present discussion applies to the mentioned superparamagnetic systems, the conclusions are general and fundamental. In many other physical situations, an Arrhenius-like switching behavior is found on the basis of assuming a temperature-independent energy barrier. In particular when this energy barrier is the result of a collective statistical behavior of many constituents, it may contain an intrinsic temperature dependence which has to be carefully taken into account, in particular when the prefactor is interpreted in terms of an attempt frequency.

\section{Acknowledgements}

We acknowledge support from the DFG Sonderforschungsbereich 668 "Magnetismus vom Einzelatom zur Nanostruktur".

\section{References}

\section{References}

[1] S. Rohart, P. Campiglio, V. Repain, Y. Nahas, C. Chacon, Y. Girard, J. Lagoute, A. Thiaville, S. Rousset, Phys. Rev. Lett. 104 (2010) 137202.

[2] A. Neumann, D. Altwein, C. Thönnißen, R. Wieser, A. Berger, A. Meyer, E. Vedmedenko, H.P. Oepen, New J. Phys. 16 (2014) 083012. 
[3] W.F. Brown Jr., J. Appl. Phys. 34 (1963) 1319.

[4] M.L. Néel, Ann. Geophys. 5 (1949) 1949.

[5] H.A. Kramers, Physica 7 (1940) 284.

[6] W.T. Coffey, Y.P.J. Kalmykov, Appl. Phys. 112 (2012) 121301.

[7] P. Hänggi, P. Talkner, M. Borkovec, Rev. Mod. Phys. 62 (1990) 251.

[8] S. Krause, G. Herzog, T. Stapelfeldt, L. Berbil-Bautista, M. Bode, E.Y. Vedmedenko, R. Wiesendanger, Phys. Rev. Lett. 103 (2009) 127202.

[9] J.L. Dormann, D. Fiorani, E. Tronc, J. Magn. Magn. Mater. 202 (1999) 251.

[10] J.L. Dormann, D. Fiorani, E. Tronc, Adv. Chem. Phys. 98 (2007) 283.

[11] H.-B. Braun, H. Neal Bertram, J. Appl. Phys. 75 (1994) 4609.

[12] H.-B. Braun, Phys. Rev. Lett. 71 (1993) 3557.

[13] U. Nowak, O.N. Mryasov, R. Wieser, K. Guslienko, R.W. Chantrell, Phys. Rev. B 72 (2005) 172410.

[14] R. Skomski, J.M.D. Coey, Phys. Rev. B 48 (1993) 15812.

[15] R. Skomski, R.D. Kirby, D.J. Sellmyer, J. Appl. Phys. 85 (1999) 5069.

[16] C. de Julián Fernández, Phys. Rev. B 72 (2005) 054438.

[17] S. Yoon, J. Magn. Magn. Mater. 324 (2012) 2620.

[18] N.A. Usov, J. Appl. Phys. 109 (2011) 023913.

[19] L. He, C. Chen, Phys. Rev. B 75 (2007) 184424.

[20] F. Wiekhorst, E. Shevchenko, H. Weller, J. Kötzler, Phys. Rev. B 67 (2003) 224416.

[21] N. Akulov, Z. Physik 100, 197 (1936).

[22] C. Zener, Phys. Rev. 96, 1335 (1954). 
[23] H.B. Callen. S. Shtrikman, Solid State Commun. 3 (1965) 5.

[24] H.B. Callen, E. Callen, J. Phys. Chem. Solids 27 (1966) 1271.

[25] W.P. Wolf, Phys. Rev. 108 (1957) 1152.

[26] R. Skomski, J. Appl. Phys. 101 (2007) 09B104.

[27] R. Yanes, O. Chubykalo-Fesenko, R.F.L. Evans, R.W. Chantrell, J. Phys. D: Appl. Phys. 43 (2010) 474009.

[28] R. Skomski, K. Pankaj, G.C. Hadjipanayis, D.J. Sellmyer, IEEE Trans. Mag. 49 (2013) 3229.

[29] A. Polewko-Klim, S. Uba, L. Uba, J. Mag. Mag. Mat. 324 (2012) 1193.

[30] T.E. Torres, E. Lima Jr., A. Mayoral, A. Ibarra, C. Marquina, M.R. Ibarra, G.F. Goya, J. Appl. Phys. 118 (2015) 183902.

[31] M. Tadic, S. Kralj, M. Jagodic, D. Hanzel, D. Makovec, Appl. Surf. Sci. 322 (2014) 255.

[32] A. Neumann, Magnetisierungsverhalten einzelner ferromagnetischer Nanostrukturen, PhD thesis, Universität Hamburg, 2014.

[33] R.J. Tackett, J.G. Parsons, B.I. Machado, S.M. Gaytan, L.E. Murr, C.E. Botez, Nanotechnology 21 (2010) 365703.

[34] O.N. Mryasov, U. Nowak, K.Y. Guslienko, R.W. Chantrell, Europhys. Lett. 69 (2005) 805.

[35] D. Böttcher, A. Ernst, J. Henk, J. Phys. Condens. Mat. 23 (2011) 296003.

[36] M. Farle, Rep. Prog. Phys. 61 (1998) 755.

[37] J. Dubowik, Phys. Rev. B 54 (1996) 1088.

[38] J.P. Pierce, M.A. Torija, Z. Gai, J. Shi, T.C. Schulthess, G.A. Farnan, J.F. Wendelken, E.W. Plummer, J. Shen, Phys. Rev. Lett. 92 (2004) 237201.

[39] U. Gradmann, Appl. Phys. 3 (1974) 161. 
[40] C.M. Schneider, P. Bressler, P. Schuster, J. Kirschner, J.J. De Miguel, R. Miranda, Phys. Rev. Lett. 64 (1990) 1059. 